\title{
REPRESENTATION THEOREMS FOR COMPLEMENTED ALGEBRAS
}

\author{
BY \\ FREDA E. ALEXANDER
}

Introduction. In this paper we obtain Hilbert space representations for as large a class of complemented algebras as possible. In [2] the same problem was considered for complemented $B^{*}$-algebras. It was shown then that if $\boldsymbol{A}$ is a topologically simple $B^{*}$-algebra, then a sufficient (subject to a dimension restriction) and a necessary condition that a complementor $p$ be expressible in terms of a Hilbert space representation of $\boldsymbol{A}$ (or, equivalently, in the form $\boldsymbol{R}^{p}=\left(\boldsymbol{R}_{l}\right)^{\#}$ for some involution \# in $\boldsymbol{A}$ ) was that $p$ be continuous. Throughout the present paper the same dimension restriction (that the algebra has no minimal left ideals of dimension less than three) will be imposed. In $\$ 5$ a counterexample shows that it cannot be removed. The definition of continuity in [2] is not applicable to a general complemented algebra, but in $\$ 2$ we give an alternative definition and show that this is an extension of the previous definition. In $\S 3$ we consider the case when $\boldsymbol{A}$ is a primitive Banach algebra. We obtain a faithful, continuous, strictly dense Hilbert space representation for $\boldsymbol{A}$ when endowed with a continuous complementor. Under this we identify $\boldsymbol{A}$ with a left ideal of $\boldsymbol{B}(\boldsymbol{H})$ that is closed under a norm that majorises the operator norm. We then show that this representation characterizes primitive Banach algebras with continuous complementors. In $\S 4$ we use the results of $\S 3$ to obtain a faithful, continuous Hilbert space representation for any semisimple Banach algebra $\boldsymbol{A}$ with a continuous complementor. Conversely, we show that, if any complemented algebra admits a representation of this form, then the complementor is continuous. We deduce that, if $A$ is $B^{*}$, then a necessary and sufficient condition that its complementor be expressible in the form $\boldsymbol{R}^{p}=\left(\boldsymbol{R}_{l}\right)^{\#}$ is that it be continuous. This extends the result of [2]. In $\S 5$ we apply the results of $\S 4$ to show that the condition $C_{2}$ in the definition of a complementor cannot be relaxed.

1. Preliminaries. Throughout the paper $\boldsymbol{A}$ will denote a semisimple complex Banach algebra whose norm is \|\|$;\left\{\boldsymbol{I}_{\lambda}: \lambda \in \Lambda\right\}$ is the set of all minimal closed two-sided ideals of $A ; R_{A}$ is the set of all closed right ideals of $A$ and $M_{A}$ the set of all minimal right ideals of $A$.

Following [8] we say that $\boldsymbol{A}$ is a right complemented algebra if there is a mapping $p: \boldsymbol{R} \rightarrow \boldsymbol{R}^{p}$ of $R_{A}$ onto itself that has the following properties:

$C_{1}: R \cap R^{p}=(0)\left(R \in R_{A}\right)$;

$C_{2}: R+R^{p}=A\left(R \in R_{A}\right)$

Received by the editors June 7, 1968.

Copyright (C) 1970, American Mathematical Society 
$C_{3}:\left(R^{p}\right)^{p}=R\left(R \in R_{A}\right) ;$

$C_{4}:$ if $\boldsymbol{R}_{1} \subset \boldsymbol{R}_{2}$ then $\boldsymbol{R}_{1}^{p} \supset \boldsymbol{R}_{2}^{p}\left(\boldsymbol{R}_{1}, \boldsymbol{R}_{2} \in R_{A}\right)$.

The mapping $p$ is called a right complementor on $\boldsymbol{A}$. A left complementor and a left complemented algebra are defined analogously. Since we shall be exclusively concerned with right complemented algebras we shall refer to them as complemented algebras and to right complementors as complementors.

We recall that, from Lemma 5 and Theorem 4 of [8], if $A$ is a complemented algebra, then $\boldsymbol{A}$ has a dense socle and is the direct topological sum of the family $\left\{\boldsymbol{I}_{\lambda}: \lambda \in \Lambda\right\}$. In particular, if $\boldsymbol{A}$ is primitive, then it is topologically simple.

The notation we adopt is mostly that of [6]; however, we follow [3] in denoting by $S_{l}, S_{r}$ respectively the left and right annihilators of a subset $S$ of an algebra. Also we use cl ( ) to denote closure.

Some other specific points of notation should be mentioned here. Let $\boldsymbol{X}$ be a Banach space. Then $[x]$ will denote the one-dimensional linear subspace of $\boldsymbol{X}$ generated by a single element $x$ of $\boldsymbol{X} . \boldsymbol{B}(\boldsymbol{X}), \boldsymbol{K}(\boldsymbol{X})$ are the algebras of all bounded, compact (respectively) linear operators on $X$. The operator norms will always be denoted by $\mid$ |. If $\boldsymbol{B}$ is a subalgebra of $\boldsymbol{B}(\boldsymbol{X})$ then:

1. $S(\boldsymbol{R})$ is the smallest closed subspace of $\boldsymbol{X}$ that contains the range of each operator in a subset $\boldsymbol{R}$ of $\boldsymbol{B}$;

2. $J_{B}(S)$ is the set of all elements in $B$ whose range is contained in a subset $\boldsymbol{S}$ of $\boldsymbol{X}$.

Suppose $\boldsymbol{S}$ and $\boldsymbol{T}$ are closed linear subspaces of $\boldsymbol{X}$ such that $\boldsymbol{S} \oplus \boldsymbol{T}=\boldsymbol{X}$. Then by Theorem 4.8.D in [7] there is a bounded linear projection operator $\boldsymbol{E}$ on $\boldsymbol{X}$ such that $x=\boldsymbol{E} x+(\mathbf{1}-\boldsymbol{E}) x$ gives the unique decomposition of an element $x$ of $\boldsymbol{X}$ into components $E x$ in $\boldsymbol{S}$ and $(\boldsymbol{1}-\boldsymbol{E}) x$ in $\boldsymbol{T}$. We write $P(\boldsymbol{S}, \boldsymbol{T})$ for $\boldsymbol{E}$. This notation is used most frequently for the operator $P\left(\boldsymbol{R}, \boldsymbol{R}^{p}\right)$ on $\boldsymbol{A}$.

The following set notations are adopted:

(1) if $\boldsymbol{A}, \boldsymbol{B}$ are subsets of an algebra then $\boldsymbol{A B}=\{a b: a \in \boldsymbol{A}, b \in \boldsymbol{B}\}$,

(2) if $\boldsymbol{S}$ is a subset of a space $\boldsymbol{X}$ and $\boldsymbol{T}$ a set of mappings of $\boldsymbol{X}$ into another space $\boldsymbol{Y}$ then $\boldsymbol{T S}=\{t s: t \in \boldsymbol{T}, s \in \boldsymbol{S}\}$,

(3) if $a \rightarrow T_{a}$ is a representation of an algebra and $A$ is a subset of the algebra then $T_{A}=\left\{T_{a}: a \in A\right\}$.

$C$ will denote the complexes and $Z$ the set of positive integers and zero.

2. Complementors and continuous complementors on $A, I_{\lambda}$. We first observe that since $\boldsymbol{A}$ is semisimple, Lemma 1 of [8] applies to $\boldsymbol{A}$; thus $\boldsymbol{I}_{\lambda}^{p}=\left(\boldsymbol{I}_{\lambda}\right)_{l}=\left(\boldsymbol{I}_{\lambda}\right)_{r}$ and, in particular, is a two-sided ideal of $\boldsymbol{A}$. Also from the proof of Lemma 2.8.8 in [6] it can be seen that every minimal right (or left) ideal of $\boldsymbol{A}$ is contained in a minimal closed two-sided ideal of $\boldsymbol{A}$. A third consequence of the semisimplicity of $\boldsymbol{A}$ is that $a \in \operatorname{cl}(a A)$ for all $a$ in $A$ (Lemma 3 in [1]).

LeMmA 2.1. If $\boldsymbol{R} \in R_{A}$ and $R \subset I_{\mu}$ then $\boldsymbol{R}^{p} \supset I_{\lambda}$ for all $\lambda \in \Lambda, \lambda \neq \mu$.

Proof. Using $C_{4}$ it is clearly sufficient to show that $\boldsymbol{I}_{\mu}^{p} \supset \boldsymbol{I}_{\lambda}(\mu \neq \lambda)$. Now $\boldsymbol{I}_{\mu}^{p} \cap \boldsymbol{I}_{\lambda}$ 
is a closed two-sided ideal of $\boldsymbol{A}$ that is contained in $\boldsymbol{I}_{\lambda}$. It must, therefore, be (0) or $\boldsymbol{I}_{\lambda}$. Suppose that there is some $\lambda \in \Lambda(\lambda \neq \mu)$ such that $\boldsymbol{I}_{\mu}^{p} \cap \boldsymbol{I}_{\lambda}=(0)$. Then let $x$, $x^{\prime}$ be any elements of $\boldsymbol{I}_{\lambda}$. Write $x=y+z$ where $y \in \boldsymbol{I}_{\mu}, \dot{z} \in \boldsymbol{I}_{\mu}^{p}$. Then $y x^{\prime} \in \boldsymbol{I}_{\mu} \cap \boldsymbol{I}_{\lambda}=(0)$ and $z x^{\prime} \in I_{\mu}^{p} \cap \boldsymbol{I}_{\lambda}=(0)$; therefore $x x^{\prime}=0$. Thus $\left(\boldsymbol{I}_{\lambda}\right)^{2}=(0)$ which contradicts the semisimplicity of $\boldsymbol{A}$. This completes the proof.

We now give the definition of continuity of a complementor $p$. It is shown at the end of this section that if $A$ is $B^{*}$ then the present definition is equivalent to that of [2].

Definition. A sequence $\left\{\boldsymbol{R}_{n}: n \in Z\right\} \subset M_{A}$ is p-convergent to $\boldsymbol{R}_{0}$ if $P\left(\boldsymbol{R}_{n}, \boldsymbol{R}_{n}^{p}\right)$ converges uniformly to $P\left(\boldsymbol{R}_{0}, \boldsymbol{R}_{0}^{p}\right)$ (as $\left.n \rightarrow \infty\right)$ on any minimal left ideal of $\boldsymbol{A}$. The complementor $p$ is continuous if whenever $a_{n} A \in M_{A}(n \in Z)$ and $a_{n} \rightarrow a_{0}$ as $n \rightarrow \infty$ then $\left\{a_{n} \boldsymbol{A}\right\}$ is $p$-convergent to $a_{0} A$.

THEOREM 2.2. Every closed right (or left) ideal of $\boldsymbol{I}_{\lambda}$ is also a closed right (left) ideal of $\boldsymbol{A}$. A complementor $p_{\lambda}$ can be induced in $\boldsymbol{I}_{\lambda}$ by $\boldsymbol{R}^{p_{\lambda}}=\boldsymbol{R}^{p} \cap \boldsymbol{I}_{\lambda}\left(\boldsymbol{R} \in \boldsymbol{R}_{\boldsymbol{I}_{\lambda}}\right)$. Further, $p$ is continuous if and only if $p_{\lambda}$ is continuous for all $\lambda$ in $\Lambda$.

Proof. Let $\boldsymbol{R}$ be any closed right ideal of $\boldsymbol{I}_{\lambda}$. Then

$$
\boldsymbol{R A}=\boldsymbol{R}\left(\boldsymbol{I}_{\lambda}+\boldsymbol{I}_{\lambda}^{p}\right)=\boldsymbol{R}\left(\boldsymbol{I}_{\lambda}+\left(\boldsymbol{I}_{\lambda}\right)_{r}\right)=\boldsymbol{R} \boldsymbol{I}_{\lambda} \subset \boldsymbol{R} .
$$

The proof for a left ideal of $\boldsymbol{I}_{\lambda}$ is similar. It is now possible to define $p_{\lambda}$ and the verification that it is a complementor is easy.

Now suppose that each $p_{\lambda}$ is continuous. Let $\left\{\boldsymbol{R}_{n}: n \in Z\right\} \subset M_{A}$. For each $n$ let $\boldsymbol{I}_{n}$ be the minimal closed two-sided ideal of $\boldsymbol{A}$ that contains $\boldsymbol{R}_{n}$. Suppose there exist elements $a_{n} \in \boldsymbol{R}_{n}$ such that $a_{n} \rightarrow a_{0} \neq 0$ as $n \rightarrow \infty$. We show first that there is some integer $N$ such that, for all $n>N, \boldsymbol{R}_{n} \subset \boldsymbol{I}_{0}$. Suppose the contrary if possible. Then, taking a subsequence if necessary, we have $a_{n} \in A, a_{n} \rightarrow a_{0}(\neq 0)$ as $n \rightarrow \infty$, and $a_{n} I_{0}=I_{0} a_{n}=(0)$. Let $t \in I_{0}:\left\|t a_{0}\right\|=\left\|t\left(a_{0}-a_{n}\right)\right\| \leqq\|t\|\left\|a_{0}-a_{n}\right\|$ and, letting $n \rightarrow \infty$, we see that $t a_{0}=0$. Thus $I_{0} a_{0}=(0)$ and hence by the topological simplicity of $\boldsymbol{I}_{0}$ we have $a_{0}=0$ which is the required contradiction. Thus we can assume that $\boldsymbol{R}_{n} \subset \boldsymbol{I}_{0}$ for all $n$ in $Z$. Then, by hypothesis $\left\{\boldsymbol{R}_{n}\right\}$ is $p_{0}$-convergent to $\boldsymbol{R}_{0}$. However, for any $R \in M_{A}$, with $R \subset I_{0}$ and any minimal left ideal $L$ of $A$ there are two possibilities (let $\boldsymbol{I}_{\lambda}$ be the minimal closed two-sided ideal of $\boldsymbol{A}$ that contains $\boldsymbol{L}$ ):

(1) $I_{0} \neq I_{\lambda}$ : then $P\left(R, R^{p}\right)=0$ on $L$ since $L \subset I_{\lambda} \subset R^{p}$,

(2) $I_{0}=I_{\lambda}$ : then $P\left(R, R^{p}\right)=P\left(R, R^{p_{0}}\right)$ on $L$.

Thus $\left\{\boldsymbol{R}_{n}\right\} p$-convergent to $\boldsymbol{R}_{0}$ is equivalent to $\left\{\boldsymbol{R}_{n}\right\} p_{0}$-convergent to $\boldsymbol{R}_{0}$. Therefore $\left\{\boldsymbol{R}_{n}\right\}$ is $p$-convergent to $\boldsymbol{R}_{0}$ and so $p$ is continuous.

The converse is now easily proved since $\left\{\boldsymbol{R}_{n}\right\} \subset M_{\boldsymbol{I}_{\lambda}}$ implies $\left\{\boldsymbol{R}_{n}\right\} \subset M_{\boldsymbol{A}}$.

Theorem 2.3. Let $R \in R_{A}$. Then $R=\operatorname{cl}\left(\sum R \cap I_{\lambda}: \lambda \in \Lambda\right)$, and

$$
\boldsymbol{R}^{p}=\operatorname{cl}\left(\sum\left(\boldsymbol{R} \cap \boldsymbol{I}_{\lambda}\right)^{p_{\lambda}}: \lambda \in \Lambda\right) .
$$

Proof. Let $a$ be any element of $R$, and let $\varepsilon>0$. Then, since $a \in \operatorname{cl}(a A)$, there is an element $b$ of $\boldsymbol{A}$ such that $\|a-a b\|<\varepsilon / 2$. Also since $\boldsymbol{A}=\operatorname{cl}\left(\sum \boldsymbol{I}_{\lambda}: \lambda \in \Lambda\right)$ there exists 
$\left\{\lambda_{i}: i=1,2, \ldots, n\right\} \subset \Lambda$ and elements $b_{i}$ of $\boldsymbol{I}_{\lambda_{i}}$ such that $\left\|b-\left(b_{1}+\cdots+b_{n}\right)\right\|$ $<\varepsilon /(2\|a\|)$. Now $\left\|a-a\left(b_{1}+\cdots+b_{n}\right)\right\|<\varepsilon$. Thus

$$
\boldsymbol{R} \subset \operatorname{cl}\left(\sum \boldsymbol{R} \boldsymbol{I}_{\lambda}: \lambda \in \Lambda\right) \subset \operatorname{cl}\left(\sum \boldsymbol{R} \cap \boldsymbol{I}_{\lambda}: \lambda \in \Lambda\right) .
$$

Conversely, $\boldsymbol{R} \supset \boldsymbol{R} \cap \boldsymbol{I}_{\lambda}$ for all $\lambda$ in $\Lambda$ and so $\boldsymbol{R} \supset \operatorname{cl}\left(\sum \boldsymbol{R} \cap \boldsymbol{I}_{\lambda}: \lambda \in \Lambda\right)$.

Now, applying this result to $\boldsymbol{R}^{p}$, we have

$$
\begin{aligned}
\boldsymbol{R}^{p} & =\operatorname{cl}\left(\sum \boldsymbol{R}^{p} \cap \boldsymbol{I}_{\lambda}: \lambda \in \Lambda\right) \subset \operatorname{cl}\left(\sum\left(\boldsymbol{R} \cap \boldsymbol{I}_{\lambda}\right)^{p} \cap \boldsymbol{I}_{\lambda}: \lambda \in \Lambda\right) \\
& =\operatorname{cl}\left(\sum\left(\boldsymbol{R} \cap \boldsymbol{I}_{\lambda}\right)^{p_{\lambda}}: \lambda \in \Lambda\right) .
\end{aligned}
$$

Also, since $\boldsymbol{R}=\operatorname{cl}\left(\sum \boldsymbol{R} \cap \boldsymbol{I}_{\lambda}: \lambda \in \Lambda\right)$ and $\boldsymbol{I}_{\lambda}^{p} \supset \boldsymbol{I}_{\mu}(\mu \neq \lambda)$, it is easily seen that $\boldsymbol{R} \subset \operatorname{cl}\left(\left(\boldsymbol{R} \cap \boldsymbol{I}_{\lambda}\right)+\boldsymbol{I}_{\lambda}^{p}\right)$. Therefore

$$
\begin{aligned}
\boldsymbol{R}^{p} \supset\left(\operatorname{cl~}\left(\left(\boldsymbol{R} \cap \boldsymbol{I}_{\lambda}\right)+\boldsymbol{I}_{\lambda}^{p}\right)\right)^{p} & \left.=\left(\boldsymbol{R} \cap \boldsymbol{I}_{\lambda}\right)^{p} \cap \boldsymbol{I}_{\lambda} \quad \text { (by Lemma } 2 \text { in [1] }\right) \\
& =\left(\boldsymbol{R} \cap \boldsymbol{I}_{\lambda}\right)^{p_{\lambda} .}
\end{aligned}
$$

Hence $\boldsymbol{R}^{p} \supset \operatorname{cl}\left(\sum\left(\boldsymbol{R} \cap \boldsymbol{I}_{\lambda}\right)^{p_{\lambda}}: \lambda \in \Lambda\right)$ and, combining this with the previous inclusion, we have equality.

Before specializing to the topologically simple case in the next section we justify our present definition of a continuous complementor by showing that, if $A$ is $B^{*}$, it is equivalent to the definition given in [2].

We recall the concepts of [2] using the notation of this paper. Suppose that $\boldsymbol{A}$ is $B^{*}$. Then a minimal idempotent $f$ of $A$ is said to be a $p$-projection if multiplication on the left by $f$ is $P\left((f A),(f A)^{p}\right)$. Every minimal right ideal of $A$ contains a unique $p$-projection as well as a unique hermitian idempotent which corresponds to the special case when $p$ is the natural complementor $\boldsymbol{R} \rightarrow\left(\boldsymbol{R}_{l}\right)^{*}$. This gives rise to the $p$-derived mapping $P$ from the set $E$ of all hermitian minimal idempotents onto the set $F$ of all $p$-projections in $A(P e=f: f A=e A)$. In [2] the complementor $p$ was said to be continuous if $P$ was continuous with respect to the relative metric topologies induced in $E, F$ by the norm in $A$.

In the special case when $\boldsymbol{A}=\boldsymbol{K}(\boldsymbol{H})$ for some Hilbert space $\boldsymbol{H}$ then, for any $\boldsymbol{x}$ in $\boldsymbol{H}$, $e_{x}, f_{x}$ denoted the unique elements of $E, F$ respectively that are contained in $J_{A}([x])$. Thus multiplication on the left by $f_{x}$ corresponds to $P\left(J_{A}([x]),\left(J_{A}([x])\right)^{p}\right)$ and by $e_{x}$ corresponds to $P\left(J_{A}([x]), J_{A}\left(x^{\perp}\right)\right)=P\left(J_{A}([x]),\left(J_{A}([x])\right)_{l}^{*}\right)$. Also $e_{x}=$ $x \otimes x /(x, x)$.

This definition is essentially a relative one in that it presupposes the existence of a natural (continuous) complementor $\boldsymbol{R} \rightarrow\left(\boldsymbol{R}_{l}\right)^{*}$. Accordingly we shall refer to it as relative continuity. In the next theorem we show that the two definitions do in fact coincide.

THEOREM 2.4. If $A$ is $B^{*}$ then $p$ is continuous if and only if it is relatively continuous.

Proof. Suppose that $p$ is continuous and that $e_{0} \in E$. If $P$ is not continuous at $e_{0}$ then there are elements $e_{n}$ of $E$ and $k>0$ such that

$$
\left\|e_{n}-e_{0}\right\|<1 / n, \quad\left\|P\left(e_{0}\right)-P\left(e_{n}\right)\right\|>k \quad(n=1,2, \ldots) .
$$


It is easy to verify that $e_{0}, e_{n}$ are contained in the same minimal closed two-sided ideal $\boldsymbol{I}$ of $\boldsymbol{A}$. Also $P\left(e_{0}\right), P\left(e_{n}\right) \in \boldsymbol{I}$. Now since any minimal left ideal of $\boldsymbol{I}$ is a minimal left ideal of $\boldsymbol{A}$ and the left regular representation of $\boldsymbol{I}$ on any minimal left ideal is an isometry, the continuity of $p$ contradicts (i).

Conversely, suppose that $p$ is relatively continuous. Let $\left\{a_{n} A: n \in Z\right\} \subset M_{A}$ be such that $a_{n} \rightarrow a_{0}$ as $n \rightarrow \infty$. Let $I_{0}$ be the minimal closed two-sided ideal of $\boldsymbol{A}$ that contains $a_{0}$; then there is an integer $N$ such that $a_{n} \in I_{0}$ for all $n>N$. (See proof of Theorem 2.2.) Also for $n>N$ :

$$
P\left(a_{n} A,\left(a_{n} A\right)^{p}\right) / I_{\lambda}=0 \quad\left(I_{\lambda} \neq I_{0}\right), \quad P\left(a_{n} A,\left(a_{n} A\right)^{p}\right) / I_{0}=P\left(a_{n} A,\left(a_{n} A\right)^{p_{0}}\right) .
$$

Thus it is sufficient to prove the result in the topologically simple case. Suppose that $\boldsymbol{A}=\boldsymbol{K}(\boldsymbol{H})$ where $\boldsymbol{H}$ is a Hilbert space with inner product ( , ) and norm \|\| . Suppose that $a_{n}=x_{n} \otimes y_{n}(n \in Z)$ and $a_{n} \rightarrow a_{0} \neq 0$ as $n \rightarrow \infty\left(x_{n}, y_{n} \in \boldsymbol{H}\right)$. We may clearly assume that for all $n$ in $Z,\left\|x_{n}\right\|=1$ and $\left|\left(y_{0}, y_{n}\right)\right|=\left(y_{0}, y_{n}\right)$. We wish to show that

$$
P\left(a_{n} A,\left(a_{n} A\right)^{p}\right)-P\left(a_{0} A,\left(a_{0} A\right)^{p}\right)
$$

tends uniformly to zero on any minimal left ideal of $\boldsymbol{A}$. This is certainly the case if $\left\|f_{x_{n}}-f_{x_{0}}\right\| \rightarrow 0$ as $n \rightarrow \infty$; by the relative continuity of $p$ the latter will follow if $\left\|e_{x_{n}}-e_{x_{0}}\right\| \rightarrow 0$ as $n \rightarrow \infty$.

We prove this. Since $\left\|a_{n}-a_{0}\right\| \rightarrow 0$, we have

Therefore

$$
\left\|\left(x_{n} \otimes y_{n}\right) y_{0}-\left(x_{0} \otimes y_{0}\right) y_{0}\right\|=\left\|\left(y_{0}, y_{n}\right) x_{n}-\left(y_{0}, y_{0}\right) x_{0}\right\| \rightarrow 0 .
$$

Now

$$
\left(y_{0}, y_{n}\right)-\left(y_{0}, y_{0}\right)=\left\|\left(y_{0}, y_{n}\right) x_{n}\right\|-\left\|\left(y_{0}, y_{0}\right) x_{0}\right\| \rightarrow 0
$$

$$
\left(y_{0}, y_{0}\right)\left\|x_{n}-x_{0}\right\| \leqq\left|\left(y_{0}, y_{0}-y_{n}\right)\right| \cdot\left\|x_{n}\right\|+\left\|\left(y_{0}, y_{n}\right) x_{n}-\left(y_{0}, y_{0}\right) x_{0}\right\| \rightarrow 0 .
$$

Thus $\left\|x_{n}-x_{0}\right\| \rightarrow 0$ and hence $\left\|e_{x_{n}}-e_{x_{0}}\right\|=\left\|x_{n} \otimes x_{n}-x_{0} \otimes x_{0}\right\| \rightarrow 0$ as $n \rightarrow \infty$.

3. The primitive case. In this section $\boldsymbol{A}$ will denote a primitive Banach algebra with a complementor $p$. Since $A$ has a dense socle it is topologically simple. (See the discussion at the beginning of $\$ 2$ in [1].)

Let $L=A e$ be a fixed minimal left ideal of $A$ and $e$ a fixed minimal idempotent. Following [1], for any closed right ideal $\boldsymbol{R}$ of $\boldsymbol{A}$ and any closed linear subspace $\boldsymbol{S}$ of $\boldsymbol{L}$, we write

$$
S(R)=R \cap L=\operatorname{cl}(R L)=R L, \quad J(S)=\operatorname{cl}(S A)=\{a \in A: a L \subset S\} .
$$

Definition. Suppose $X$ is a Banach space and $S \rightarrow S^{q}$ a map of the set $S$ of all closed linear subspaces of $\boldsymbol{X}$ onto itself that has the following properties:

$$
\begin{aligned}
& L_{1}: S \cap S^{q}=(0)(S \in S) \\
& L_{2}: S+S^{q}=X(S \in S) \\
& L_{3}:\left(S^{q}\right)^{q}=S(S \in S) ; \\
& L_{4}: \text { if } S_{1} \subset S_{2} \text { then } S_{1}^{q} \supset S_{2}^{q}\left(S_{1}, S_{2} \in S\right) .
\end{aligned}
$$


Then $q: S \rightarrow S^{q}$ is a linear space complementor (LSC) on $\boldsymbol{X}$. The LSC is continuous if $P\left(\left[x_{n}\right],\left[x_{n}\right]^{q}\right)$ converges uniformly to $P\left(\left[x_{0}\right],\left[x_{0}\right]^{q}\right)$ whenever $\left\{x_{n}: n \in Z\right\} \subset X$ and $x_{n} \rightarrow x_{0} \neq 0$.

Now it can be shown using [8] (in particular Lemma 6 and corollary to Lemma $10)$ that the maps $J, S$ are one-to-one, that $J$ is the inverse of $S$, and hence that $S \rightarrow S^{q}=S\left((J(S))^{p}\right)$ is an LSC on $L$. (See the discussion at the beginning of $\S 2$ in [1].)

THEOREM 3.1. The map $q$ is an LSC on L. If $p$ is continuous then $q$ is continuous.

Proof. The first part is discussed above. Now suppose that $p$ is continuous. Let $\left\{x_{n}\right\}$ be a sequence in $\boldsymbol{L}$ that converges to a nonzero element $x_{0}$ of $\boldsymbol{L}$. Then for $n$ in $Z$, $x_{n} A \in M_{A}$. Let $E_{n}$ be $P\left(\left(x_{n} A\right),\left(x_{n} A\right)^{p}\right)$. Since $x_{n} \in \operatorname{cl}\left(x_{n} A\right)=x_{n} A$ and $p$ is continuous, $\left\{E_{n}\right\}$ converges uniformly to $E_{0}$ on $\boldsymbol{L}$. Now for any $z$ in $\boldsymbol{L}$ :

$$
E_{n} z+\left(1-E_{n}\right) z=z=z e=E_{n} z e+\left(1-E_{n}\right) z e
$$

and here $E_{n} z, E_{n} z e \in x_{n} A$ and $\left(1-E_{n}\right) z,\left(1-E_{n}\right) z e \in\left(x_{n} A\right)^{p}$. Thus, by $C_{2}, E_{n} z=E_{n} z e$, and so $E_{n} z \in x_{n} A \cap \boldsymbol{L}=S\left(x_{n} A\right)=\left[x_{n}\right]$. Similarly $\left(1-E_{n}\right) z \in\left[x_{n}\right]^{q}$. Therefore, $E_{n} / \boldsymbol{L}=P\left(\left[x_{n}\right],\left[x_{n}\right]^{q}\right)$. It is now immediate that $q$ is continuous.

COROllaRY. An inner product (, ) can be induced into $L$. With this inner product $\boldsymbol{L}$ becomes a Hilbert space with norm equivalent to its original norm.

Proof. This is an immediate consequence of Theorem 2 of [4].

We now consider the algebra $\boldsymbol{F}=\boldsymbol{K}(\boldsymbol{L})$. By the above corollary $\boldsymbol{F}$, under an equivalent norm, is a dual $B^{*}$-algebra. Via the left regular representation on $\boldsymbol{L}$, $\boldsymbol{A}$ can be imbedded in $\boldsymbol{F}$. We now show that $\boldsymbol{F}$ has a complementor $p_{e}$ that is a natural extension of $p$.

TheOREM 3.2. A complementor $p_{e}$ is induced in $\boldsymbol{F}$ by $p$. If $p$ is continuous then $p_{e}$ is continuous.

Proof. For any $R \in R_{F}$ define $R^{p_{e}}$ to be $J_{F}\left((S(R))^{q}\right)$. It is clear that $p_{e}$ maps $R_{\boldsymbol{F}}$ into itself and satisfies $C_{1}, C_{4}$. Now for any $R \in R_{F}$ and $a$ in $F$ write $P$ for $P\left(S(R),(S(R))^{q}\right)$. Then $a=\lim _{n \rightarrow \infty} a_{n}$ where $\left\{a_{n}\right\}$ is a sequence of operators of finite rank on $L$. Thus $P a_{n} \in F$. Also $\left\{P a_{n}\right\}$ is Cauchy and so converges to an element $b$ of $\boldsymbol{F}$. From Theorem 18 in [3] $P a_{n} \in \boldsymbol{R}$, and, since $\boldsymbol{R}$ is closed, $b \in \boldsymbol{R}$. Now by a similar argument $\left\{(1-P) a_{n}\right\}$ converges to an element $c$ of $\boldsymbol{R}^{p_{e}}$. Then $a=b+c \in \boldsymbol{R}+\boldsymbol{R}^{p_{e}}$. Since $a$ and $R$ were arbitrary, this proves $C_{2}$. Finally, by another application of Theorem 18 in [3], $R=J_{F}(S(R))\left(R \in R_{F}\right)$ and thus $p_{e}$ satisfies $C_{3}$.

Now suppose that $p$ is continuous. Let $\left\{a_{n}\right\}$ be a sequence of operators of rank one on $L$ such that $a_{n} \rightarrow a_{0} \neq 0$ as $n \rightarrow \infty$. Then we wish to show that $\left\{a_{n} F\right\}$ is $p_{e}$-convergent to $a_{0} \boldsymbol{F}$. Every maximal right ideal in $\boldsymbol{F}$ is modular (since $\boldsymbol{F}$ is dual) and so by Corollary 3.3 in [2] every minimal right ideal in $\boldsymbol{F}$ contains a unique $p_{e}$-projection. Let $f_{n}$ be the $p_{e}$-projection satisfying $f_{n} \boldsymbol{F}=a_{n} \boldsymbol{F}(n \in Z)$. Then it is 
clearly sufficient to show that $\left|f_{n}-f_{0}\right| \rightarrow 0$ as $n \rightarrow \infty$. However, in the verification of $C_{2}$ for $p_{e}$ it was shown that for $a \in F, R \in R_{F}$

$$
a=P a+(1-P) a, \quad P=P\left(S(R),(S(R))^{q}\right)
$$

is the decomposition of $a$ into its components in $\boldsymbol{R}, \boldsymbol{R}^{p_{e}}$. Since we know this decomposition to be unique we may deduce that $f_{n}$ is $P\left(a_{n} L,\left(a_{n} L\right)^{q}\right)$. Now by Theorem 3.1, $q$ is continuous and so $\left|f_{n}-f_{0}\right| \rightarrow 0$ as $n \rightarrow \infty$ and the proof is complete.

Thus when $p$ is continuous $F$ is, under an equivalent norm, a dual $B^{*}$-algebra with a continuous complementor $p_{e}$. Thus the work of [2] is applicable to $\boldsymbol{F}$. We recall some notation of [2].

Notation. Let $\boldsymbol{H}$ be a Hilbert space, $\boldsymbol{F}$ be $\boldsymbol{K}(\boldsymbol{H})$, and $\langle,>$ any equivalent inner product in $\boldsymbol{H}$ (i.e., one that gives rise to a norm that is equivalent to the given norm in $\boldsymbol{H})$. Then $p_{\langle\rangle}$denotes the complementor $\boldsymbol{R} \rightarrow J_{F}\left(\left(S(R)^{\perp}\langle\rangle\right)=\left(\boldsymbol{R}_{l}\right)^{*}\langle\rangle\right.$ (where ${ }^{\perp}\langle\rangle,{ }^{*}\langle\rangle$ denote respectively the orthogonal complement and the adjoint with respect to $\langle$,$\rangle ). For the proof that this a complementor and that the two$ expressions for it are the same see Corollary 4.3 in [2].

Now Theorem 6.11 of [2] gives the following.

THEOREM 3.3. If $p$ is continuous and the dimension of $\boldsymbol{L}$ is at least three then an equivalent inner product $\langle$,$\rangle can be introduced into L$ such that $p_{e}=p_{\langle\rangle}$. The LSC $q$ in $\boldsymbol{L}$ corresponds to orthogonal complementation with respect to $\langle$,$\rangle .$

We can now prove the main result of this section.

THEOREM 3.4. Let A be a primitive Banach algebra with a continuous complementor $p$ and with no left ideals of dimension less than three. Then $A$ has a faithful, continuous, strictly dense representation $a \rightarrow T_{a}$ on a Hilbert space $H$. Also:

(1) $R^{p}=\left\{a \in A: T_{a} H \perp T_{R} H\right\}\left(R \in R_{A}\right)$;

(2) the socle of $\boldsymbol{A}$ consists of all elements of $\boldsymbol{A}$ whose image is of finite rank on $\boldsymbol{H}$. This image is generated by the set of all operators $x \otimes y$ where $x$ ranges through $\boldsymbol{H}$ and $y$ ranges through a dense subspace $\boldsymbol{H}_{0}$ of $\boldsymbol{H}$;

(3) $T_{\boldsymbol{A}}$ is a left ideal of $\boldsymbol{B}(\boldsymbol{H})$.

Proof. Let $L=A e$ be a given minimal left ideal of $\boldsymbol{A}$ ( $e$ is a minimal idempotent) and $\langle,>$ the inner product induced in $L$ by Theorem 3.3. Let $H$ be the resultant Hilbert space and let $a \rightarrow T_{a}$ be the representation of $\boldsymbol{A}$ on $\boldsymbol{H}$ corresponding to the left regular representation of $\boldsymbol{A}$ on $\boldsymbol{L}$. Since $\boldsymbol{A}$ is primitive and $\boldsymbol{L}$ is minimal $a \rightarrow T_{a}$ is faithful, continuous, and strictly dense. Properties (1), (2) and that $E T_{A} \subset T_{A}$ for any orthogonal projection $E$ on $H$ are proved exactly as in Theorem 1 of [1] (for Theorem 3.3 gives $(\boldsymbol{R} L)^{\perp}=(\boldsymbol{R} L)^{q}=\left(\boldsymbol{R}^{p} \boldsymbol{L}\right)$ for any $\boldsymbol{R}$ in $\boldsymbol{R}_{\boldsymbol{A}}$; the infinite dimensionality of $\boldsymbol{A}$ was used in [1] solely to establish this). By Theorem 1 in [5] any element of $\boldsymbol{B}(\boldsymbol{H})$ is a finite linear combination of orthogonal projections; (3) is now immediate.

Note. We say that the inner product $\langle,>$ in $L$ represents $p$. 
THeOREM 3.5. Let $\boldsymbol{H}$ be a Hilbert space and $\boldsymbol{A}$ a strictly dense subalgebra of $\boldsymbol{K}(\boldsymbol{H})$. Suppose that $A$ is a Banach algebra under a norm \| \| that majorises the operator norm | |. Then $\boldsymbol{A}$ has a continuous complementor if it is a left ideal of $\boldsymbol{B}(\boldsymbol{H})$.

Proof. Theorem 3 in [1] states that a complementor $p$ is defined in $\boldsymbol{A}$ by $\boldsymbol{R}^{p}$ $=J_{A}\left((S(R))^{\perp}\right)\left(R \in R_{A}\right)$. We show that $p$ is continuous. Let $\left\{R_{n}: n \in Z\right\} \subset M_{A}$ and suppose there are elements $a_{n} \in \boldsymbol{R}_{n}$ such that, in $A, a_{n} \rightarrow a_{0} \neq 0$ as $n \rightarrow \infty$. Let $E_{n}$ be $P\left(\boldsymbol{R}_{n}, \boldsymbol{R}_{n}^{p}\right)$. Since \|\| majorises ||$,\left|a_{n}-a_{0}\right| \rightarrow 0$. Let $\boldsymbol{F}$ be $\boldsymbol{K}(\boldsymbol{H})$; then the natural complementor $\boldsymbol{R} \rightarrow\left(\boldsymbol{R}_{l}\right)^{*}$ in $\boldsymbol{F}$ is continuous (by Theorem 2.4 and the definition of relative continuity). Let $S_{n}$ be $S\left(R_{n}\right)$ and $P_{n}$ be $P\left(S_{n}, S_{n}^{\perp}\right)$. As in the proof of Theorem 3.2, it can be verified that left multiplication by $P_{n}$ is $P\left(a_{n} F,\left(a_{n} F\right)_{l}^{*}\right)$ and $P\left(a_{n} A,\left(a_{n} A\right)^{p}\right)$. By the continuity of $R \rightarrow\left(R_{l}\right)^{*}$ in $F,\left|P_{n}-P_{0}\right| \rightarrow 0$.

Now let $L$ be any minimal left ideal of $A$. Then

$$
L=\{x \otimes g: g \in \boldsymbol{H} \text { fixed, } x \in \boldsymbol{H} \text { variable }\}
$$

and by Lemma 2.4.13 in [6] the map $x \otimes g \rightarrow x$ is a bicontinuous isomorphism of $L$ onto $H$. Under this map $E_{n}(x \otimes g) \rightarrow P_{n} x$. It is thus clear that $\left\{E_{n}\right\}$ converges uniformly to $E_{0}$ on $L$. Thus $p$ is continuous.

4. General representation theorems. Throughout this section $\boldsymbol{A}$ will denote a semisimple Banach algebra with a continuous complementor $p .\left\{\boldsymbol{I}_{\lambda}: \lambda \in \Lambda\right\}$ is the set of all minimal closed two-sided ideals of $\boldsymbol{A}$ and $p_{\lambda}$ denotes the continuous complementor induced in $\boldsymbol{I}_{\boldsymbol{\lambda}}$ by $\boldsymbol{p}$.

Notation. $\boldsymbol{R}_{\lambda}$ will denote $\boldsymbol{R} \cap \boldsymbol{I}_{\lambda}\left(\boldsymbol{R} \in R_{A}, \lambda \in \Lambda\right)$. $E_{\boldsymbol{R}}$ will denote $P\left(\boldsymbol{R}, \boldsymbol{R}^{p}\right)$ $\left(R \in R_{A}\right)$ and $E_{\lambda}$ will denote $E_{I_{\lambda}}$.

THEOREM 4.1. $E_{R_{\lambda}}=E_{\lambda} \cdot E_{R}$ and $E_{\lambda}\left(1-E_{R}\right)=\left(1-E_{R_{\lambda}}\right) E_{\lambda}\left(R \in R_{A}, \lambda \in \Lambda\right)$.

Proof. Let $a \in A$. Then:

$$
E_{\lambda} a=E_{\lambda}\left(E_{R} a+\left(1-E_{R}\right) a\right)=E_{\lambda} E_{R} a+E_{\lambda}\left(1-E_{R}\right) a .
$$

Also:

$$
E_{\lambda} a=E_{R_{\lambda}} E_{\lambda} a+\left(1-E_{R_{\lambda}}\right) E_{\lambda} a .
$$

Now, by Lemma 2.1, $E_{\lambda}\left(\sum_{\mu \in \Lambda} \boldsymbol{R}_{\mu}\right)=\boldsymbol{R}_{\lambda}$ and by Theorem $2.3 \sum_{\mu \in \Lambda} \boldsymbol{R}_{\mu}$ is a dense subspace of $R=E_{R} A$. Thus, since $E_{\lambda}$ is continuous and $R_{\lambda}$ is closed, we have $E_{\lambda}\left(E_{R_{\lambda}}\right)=R_{\lambda}$. Similarly, $E_{\lambda}\left(\left(1-E_{R}\right) A\right)=R_{\lambda}^{p_{\lambda}}$. Therefore $E_{\lambda} E_{R} a, E_{R_{\lambda}} E_{\lambda} a \in R_{\lambda}$ and $E_{\lambda}\left(1-E_{R}\right) a,\left(1-E_{R_{\lambda}}\right) E_{\lambda} a \in R_{\lambda}^{p_{\lambda}}$. Thus by $C_{1}$ for $p$, the expressions (1), (2) are identical. Hence $E_{\lambda}\left(1-E_{R}\right) a=\left(1-E_{R_{\lambda}}\right) E_{\lambda} a$ and

$$
E_{\lambda} E_{R} a=E_{R_{\lambda}} E_{\lambda} a=E_{R_{\lambda}}\left(a-\left(1-E_{\lambda}\right) a\right)=E_{R_{\lambda}} a .
$$

Corollary. For each $\lambda \in \Lambda$ select $U_{\lambda}$ such that $U_{\lambda} A \in M_{A}, U_{\lambda} A \subset I_{\lambda}$, and $U_{\lambda}=P\left(U_{\lambda} A,\left(U_{\lambda} A\right)^{p}\right)$. Then $\sup \left\{\left|U_{\lambda}\right| I_{\lambda} \mid: \lambda \in \Lambda\right\}<\infty$. 
Proof. Let $R=\operatorname{cl}\left(\sum_{\lambda \in \Lambda} U_{\lambda} A\right)$; then $R \in R_{A}$ and so $E_{R}$ is a bounded linear operator on $A$. Now let $a \in I_{\lambda} ; a=E_{R_{\lambda}} a+\left(1-E_{R_{\lambda}}\right) a$ and thus, in particular, $\left(1-E_{\boldsymbol{R}_{\lambda}}\right) a \in \boldsymbol{I}_{\lambda}$. Therefore, $\left(1-E_{\boldsymbol{R}_{\lambda}}\right) a \in \boldsymbol{I}_{\lambda} \cap \boldsymbol{R}_{\lambda}^{p}=\boldsymbol{R}_{\lambda}^{p_{\lambda}} \subset \boldsymbol{R}^{p}$. Since $E_{\boldsymbol{R}_{\lambda}} a \in \boldsymbol{R}_{\lambda} \subset \boldsymbol{R}, \boldsymbol{C}_{1}$ for $p$ gives $E_{R_{\lambda}} a=E_{R} a$ or $E_{R} / I_{\lambda}=E_{R_{\lambda}} / I_{\lambda}$.

Also, $R_{\lambda}=E_{R_{\lambda}} A=E_{\lambda} E_{R} A=E_{\lambda}\left(\operatorname{cl}\left(\sum_{\lambda \in \Lambda} U_{\mu} A\right)\right)=U_{\lambda} A$. Therefore $E_{R_{\lambda}}=U_{\lambda}$. Hence $E_{R} / I_{\lambda}=U_{\lambda} / I_{\lambda}$ and so $\left|U_{\lambda} / I_{\lambda}\right| \leqq\left|E_{R}\right|$.

The following lemma shows that the inner products representing the complementors $p_{\lambda}$ can be chosen to be uniformly equivalent to the norms on minimal left ideals of $\boldsymbol{I}_{\lambda}$. This is essential for the construction of a representing Hilbert space for $A$.

Lemma 4.3. Suppose that $A$ has no minimal left ideals of dimension less than three. Then we can select minimal left ideals $L_{\lambda}$ in $I_{\lambda}$ and induce inner products $\langle,\rangle_{\lambda}$ in $L_{\lambda}$ such that $\langle,\rangle_{\lambda}$ represents $p_{\lambda}$ and for some finite constant $M$

$$
\|x\|^{2} \leqq\langle x, x\rangle_{\lambda} \leqq M^{2}\|x\|^{2} \quad\left(x \in L_{\lambda}, \lambda \in \Lambda\right) .
$$

Proof. Since any minimal left ideal in $\boldsymbol{I}_{\lambda}$ is a minimal left ideal in $\boldsymbol{A}$ its dimension is at least three. So we may select minimal left ideals $\boldsymbol{L}_{\lambda}$ of $\boldsymbol{I}_{\lambda}$ and in them induce inner products $\langle,\rangle_{\lambda}$ representing $p_{\lambda}$ as in Theorem 3.3. The inner product norm ||$_{\lambda}$ is equivalent to the original norm in $\boldsymbol{L}_{\lambda}$ and, since $\langle,\rangle_{\lambda}$ retains the above properties on multiplication by a positive real constant we may suppose

$$
\|x\| \leqq|x|_{\lambda} \nsucceq \sqrt{ } 2\|x\| \quad\left(x \in L_{\lambda}, \lambda \in \Lambda\right) .
$$

Suppose that it is not now possible to find $M$. Then there exists a sequence $\left\{\lambda_{n}\right\} \subset \Lambda$ such that ||$_{\lambda_{n}} \nless n\|\|$ on $L_{\lambda}$. For convenience we shall now replace the suffices $\lambda_{n}$ by $n$. Then there are elements $x_{n}$ of $L_{n}$ such that $\left\|x_{n}\right\|=1$, and $\left\langle x_{n}, x_{n}\right\rangle_{n}$ $=k_{n}^{2}>n^{2}$. Also, by (1), there are elements $z_{n}$ of $L_{n}$ such that $\left\|z_{n}\right\|=1,\left\langle z_{n}, z_{n}\right\rangle_{n} \leqq 2$. Now write $z_{n}=\alpha_{n} x_{n}+x_{n}^{\prime}$ where $\left\langle x_{n}, x_{n}^{\prime}\right\rangle_{n}=0, \alpha_{n} \in C$. Then:

$$
\left\langle z_{n}, z_{n}\right\rangle_{n}=\left|\alpha_{n}\right|^{2} k_{n}^{2}+\left\langle x_{n}^{\prime}, x_{n}^{\prime}\right\rangle_{n} \text {. }
$$

From (2) we deduce:

(3) $\left\langle x_{n}^{\prime}, x_{n}^{\prime}\right\rangle_{n} \leqq 2$;

(4) $\left|\alpha_{n}\right| \leqq \sqrt{ } 2 / k_{n}<\sqrt{ } 2 / n$.

Also, since $\left\|z_{n}\right\|+\left|\alpha_{n}\right| \cdot\left\|x_{n}\right\| \geqq\left\|x_{n}^{\prime}\right\| \geqq\left\|z_{n}\right\|-\left|\alpha_{n}\right| \cdot\left\|x_{n}\right\|$, we have from (4)

$$
1+\sqrt{ } 2 / n>\left\|x_{n}^{\prime}\right\|>1-\sqrt{ } 2 / n \text {. }
$$

We shall consider the subspace $\left[y_{n}\right]$ of $L_{n}$ where $y_{n}=\left(1 / k_{n}\right) x_{n}+x_{n}^{\prime}$. Let $\boldsymbol{R}_{n}$ be $J_{I_{n}}\left(\left[y_{n}\right]\right)$. Then $\boldsymbol{R}_{n} \in M_{I_{n}} \subset M_{A}$; let $U_{n}$ be $P\left(\boldsymbol{R}_{n}, \boldsymbol{R}_{n}^{p}\right)$. Then by the corollary to Theorem 4.1, there exists a finite constant $M$ such that $\left|U_{n}\right| I_{n} \mid<M$. However, $P\left(\left[y_{n}\right], y_{n}^{\perp_{n}}\right)$ is $\left(y_{n} \otimes_{n} y_{n}\right) /\left\langle y_{n}, y_{n}\right\rangle_{n}$. (We use $\perp_{\lambda}, \otimes_{\lambda}$ to denote orthogonality and tensor product respectively with respect to $\langle,\rangle_{\lambda}$.) Also, from the proof of Theorem 3.1, $U_{n} / L_{n}=P\left(\left[y_{n}\right], y_{n}^{\frac{1}{n}}\right)$. Therefore

(6) $\quad M \geqq\left|U_{n}\right| I_{n}|\geqq| U_{n}\left|L_{n}\right| \geqq\left\|U_{n} x_{n}\right\|=\left\|\frac{y_{n} \otimes_{n} y_{n}}{\left\langle y_{n}, y_{n}\right\rangle_{n}} x_{n}\right\|=\frac{\left|\left\langle x_{n}, y_{n}\right\rangle_{n}\right|}{\left\langle y_{n}, y_{n}\right\rangle_{n}}\left\|y_{n}\right\|$. 
However, from the definition of $y_{n}$ and (3), (5) we have

(7) $\left\langle y_{n}, y_{n}\right\rangle_{n}=1+\left\langle x_{n}^{\prime}, x_{n}^{\prime}\right\rangle_{n} \leqq 3$;

(8) $\left\|y_{n}\right\| \geqq\left\|x_{n}^{\prime}\right\|-\left\|\left(1 / k_{n}\right) x_{n}\right\|>1-\sqrt{ } 2 / n-1 / n$;

(9) $\left\langle x_{n}, y_{n}\right\rangle_{n}=k_{n}>n$.

Substituting (7), (8), (9) in (6): $M>(n / 3)(1-\sqrt{ } 2 / n-1 / n)$ for all $n$ which is the required contradiction.

THEOREM 4.4. Let $\boldsymbol{A}$ be a semisimple Banach algebra with a continuous complementor $p$. Suppose that $\boldsymbol{A}$ has no minimal left ideals of dimension less than three. Then $\boldsymbol{A}$ has a faithful, continuous representation $a \rightarrow T_{a}$ on a Hilbert space $\boldsymbol{H}$. For any closed right ideal $\boldsymbol{R}$ of $\boldsymbol{A},\left(T_{\boldsymbol{R}} \boldsymbol{H}\right)^{\perp}=\operatorname{cl}\left(T_{\boldsymbol{R}^{p}} \boldsymbol{H}\right) ; \boldsymbol{R}^{p}=\left\{a \in A: T_{a} \boldsymbol{H} \perp T_{\boldsymbol{R}} \boldsymbol{H}\right\}$.

Proof. Select minimal left ideals $\boldsymbol{L}_{\lambda}$ of $\boldsymbol{I}_{\lambda}$ and induce an inner product $\langle,\rangle_{\lambda}$ in $\boldsymbol{L}_{\lambda}$ as in Lemma 4.3. Let $\boldsymbol{H}_{\lambda}$ be the resultant Hilbert space for each $\lambda$ in $\Lambda$. We shall construct the direct sum of the representations $a \rightarrow T_{a}^{\lambda}$ of $\boldsymbol{A}$ on $\boldsymbol{H}_{\lambda}$ corresponding to the left regular representations of $\boldsymbol{A}$ on $\boldsymbol{L}_{\lambda}$. Let $\boldsymbol{H}=\sum^{(2)} \boldsymbol{H}_{\lambda}$ in the notation of [6, p. 197]. For each $a$ in $\boldsymbol{A}$ define $T_{a}$ by $\left(T_{a} f\right)(\lambda)=T_{a}^{\lambda} f(\lambda)(f \in \boldsymbol{H})$. Then $\left\|T_{a}^{\lambda} f(\lambda)\right\| \leqq\|a\|\|f(\lambda)\| \leqq\|a\||f(\lambda)|_{\lambda}$, and thus

$$
\sum_{\lambda \in \Lambda}\left|\left(T_{a} f\right)(\lambda)\right|^{2} \leqq M^{2} \sum_{\lambda \in \Lambda}\left\|\left(T_{a} f\right)(\lambda)\right\|^{2} \leqq M^{2} \sum_{\lambda \in \Lambda}\|a\|^{2}|f(\lambda)|_{\lambda}^{2}=M^{2}\|a\|^{2}|f|^{2} .
$$

Therefore, $T_{a} f \in \boldsymbol{H}$ for all $f$ in $\boldsymbol{H}$ and $\boldsymbol{a}$ in $\boldsymbol{A}$ and so the direct sum of the representations is defined and $\left|T_{a}\right| \leqq M\|a\|(a \in A)$, so that the representation is continuous.

Let $\boldsymbol{R} \in \boldsymbol{R}_{\boldsymbol{A}}$. Then $\boldsymbol{R}=\mathrm{cl}\left(\sum_{\lambda \in \Lambda} \boldsymbol{R}_{\lambda}\right), \boldsymbol{R}^{p}=\mathrm{cl}\left(\sum_{\lambda \in \Lambda} \boldsymbol{R}_{\lambda \lambda}^{p_{\lambda}}\right)$ and, since the representation is continuous, $\left\{h \in T_{a} H ; a \in \sum_{\lambda \in \Lambda} R_{\lambda}\right\}$ and $\left\{h \in T_{a} H: a \in \sum_{\lambda \in \Lambda} R_{\lambda}^{p_{\lambda}}\right\}$ are dense subspaces of $T_{R} H, T_{R^{p}} H$ respectively. Thus:

$$
\begin{aligned}
\left(T_{\boldsymbol{R}} \boldsymbol{H}\right)^{\perp} & =\left\{h \in T_{a} \boldsymbol{H}: a \in \sum_{\lambda \in \Lambda} \boldsymbol{R}_{\lambda}\right\}^{\perp}=\left\{h: h(\lambda) \in S\left(\boldsymbol{R}_{\lambda}\right)^{\perp}\right\}=\left\{h: h(\lambda) \in S\left(\boldsymbol{R}_{\lambda}^{p_{\lambda}}\right)\right\} \\
& =\operatorname{cl}\left\{h \in T_{a} \boldsymbol{H}: a \in \sum_{\lambda \in \Lambda} \boldsymbol{R}_{\lambda^{\lambda}}^{p}\right\}=\operatorname{cl}\left(\boldsymbol{T}_{\boldsymbol{R}^{p}} \boldsymbol{H}\right) .
\end{aligned}
$$

Next, we show that the representation $a \rightarrow T_{a}$ is faithful. Let $a \in A$; then $a \in \operatorname{cl}(a \boldsymbol{A})$ which we shall denote by $\boldsymbol{R}$. Then $\boldsymbol{R}_{\lambda}=\operatorname{cl}\left(\left(E_{\lambda} a\right) \boldsymbol{I}_{\lambda}\right)$ : in fact $\left(E_{\lambda} a\right) \boldsymbol{I}_{\lambda}$ $\subset E_{\lambda} E_{\boldsymbol{R}} \boldsymbol{A}=E_{\boldsymbol{R}_{\lambda}} \boldsymbol{A}=\boldsymbol{R}_{\lambda}$ and so $\mathrm{cl}\left(\left(E_{\lambda} a\right) \boldsymbol{I}_{\lambda}\right) \subset \boldsymbol{R}_{\lambda}$; conversely, given $\lambda \in \Lambda, b \in \boldsymbol{R}_{\lambda}$, $\varepsilon>0$, there exists $c \in A$ such that $\|b-a c\|<\varepsilon /\left|E_{\lambda}\right|$ and then $\left\|b-E_{\lambda} a c\right\|<\varepsilon$. Suppose $T_{a}=0$; then $T_{E_{\lambda} a}^{\lambda}=T_{a}^{\lambda}=0$ and, since $a \rightarrow T_{a}^{\lambda}$ is faithful on $I_{\lambda}$, we have $E_{\lambda} a=0$. Therefore, $\boldsymbol{R}_{\lambda}=\operatorname{cl}\left(E_{\lambda} a I_{\lambda}\right)=(0)$ and $\boldsymbol{R}=\operatorname{cl}\left(\sum_{\lambda \in \Lambda} \boldsymbol{R}_{\lambda}\right)=(0)$. It follows that $a=0$.

Finally we show that if $T_{a} H \subset\left(T_{R} H\right)^{\perp}$ then $a \in R^{p}$. Given such an $a$, put $a=a_{1}+a_{2}$ where $a_{1} \in \boldsymbol{R}, a_{2} \in \boldsymbol{R}^{p}$. Then for any $h$ in $\boldsymbol{H}: T_{a} h=T_{a_{1}} h+T_{a_{2}} h$. Thus, since $\left(T_{\boldsymbol{R}} \boldsymbol{H}\right)^{\perp}$ $\supset T_{R^{p}} H, T_{a_{1}} h \in T_{R} H \cap\left(T_{R} H\right)^{\perp}=(0)$. Therefore, $T_{a_{1}}=0$ and, since the representation is faithful, $a_{1}=0$ and $a=a_{2} \in \boldsymbol{R}^{p}$.

Note. In this case we cannot have $T_{A}$ a left ideal of $B(H)$. Let $\mu, \rho$ be distinct elements of $\Lambda$. Let $x, y$ be elements of $\boldsymbol{H}$ that satisfy $x(\rho)=0, y(\rho) \neq 0, x(\mu)=y(\mu) \neq 0$ 
(=h, say). Then for any $a$ in $A\left(T_{a} x\right)(\rho)=T_{a}^{\rho} x(\rho)=0$. However, since $T_{I_{\mu}}$ contains all operators of finite rank on $H$, there exists $b$ in $I_{\mu}$ such that $T_{b}^{\mu}=h \otimes_{\mu} h$. Consider $y \otimes y \cdot T_{b} x:$

$$
\begin{aligned}
\left(y \otimes y \cdot T_{b} x\right)(\rho) & =\left(y, T_{b} x\right) y(\rho)=\left(y(\mu), T_{b}^{\mu} x(\mu)\right) \cdot y(\rho) \\
& =(h, h)^{2} \cdot y(\rho) \neq 0 .
\end{aligned}
$$

Therefore, there does not exist $a$ in $A$ such that $y \otimes y \cdot T_{b}=T_{a}$.

THEOREM 4.5. Let $\boldsymbol{A}$ be a semisimple algebra of operators on a Hilbert space $\boldsymbol{H}$ that is a Banach algebra under a norm \| $\|$ that majorises the operator norm | |. Suppose $\boldsymbol{R} \rightarrow \boldsymbol{R}^{p}=J_{A}\left((\boldsymbol{R H})^{\perp}\right)\left(\boldsymbol{R} \in R_{A}\right)$ is a complementor on $\boldsymbol{A}$. Then $p$ is continuous.

Proof. Let $\left\{\boldsymbol{I}_{\lambda}: \lambda \in \Lambda\right\}$ be the set of all minimal closed-two sided ideals of $\boldsymbol{A}$. Then, since $\boldsymbol{A}$ is complemented, $\boldsymbol{A}=\mathrm{cl}\left(\sum_{\lambda \in \Lambda} I_{\lambda}\right)$; also a complementor $p_{\lambda}$ is induced in each $\boldsymbol{I}_{\lambda}$. By Theorem 2.2 it is sufficient to show that each $p_{\lambda}$ is continuous. Let $\boldsymbol{I}_{\lambda} \boldsymbol{H}=\boldsymbol{H}_{\lambda}$, and for any set $\boldsymbol{S}$ in $\boldsymbol{H}$ let $\boldsymbol{S}^{\perp_{\lambda}}=\left\{x \in \boldsymbol{H}_{\lambda}: x \perp \boldsymbol{S}\right\}$. If $\boldsymbol{a} \in \boldsymbol{A}$ and $\boldsymbol{a H} \boldsymbol{H} \subset \boldsymbol{H}_{\lambda}$ then $a \in \boldsymbol{I}_{\lambda}$ : in fact, put $a=a_{1}+a_{2}$ where $a_{1} \in \boldsymbol{I}_{\lambda}, a_{2} \in \boldsymbol{I}_{\lambda}^{p}$, then $a_{2} \boldsymbol{H} \subset \boldsymbol{H}_{\lambda} \cap \boldsymbol{H}_{\lambda}^{\perp}=(0)$ and thus $a_{2}=0$. Let $R \in R_{I_{\lambda}}$; then

$$
\begin{aligned}
\boldsymbol{R}^{p_{\lambda}} & =\boldsymbol{R}^{p} \cap \boldsymbol{I}_{\lambda}=\left\{a: a \boldsymbol{H} \subset(\boldsymbol{R} \boldsymbol{H})^{\perp}, a \in \boldsymbol{I}_{\lambda}\right\}=\left\{a: a \boldsymbol{H} \subset(\boldsymbol{R} \boldsymbol{H})^{\perp}{ }_{\lambda}\right\} \\
& =\left\{a: a \boldsymbol{H}_{\lambda} \subset\left(\boldsymbol{R} \boldsymbol{H}_{\lambda}\right)^{\perp}\right\}
\end{aligned}
$$

and so, from the proof of Theorem $3.5, p_{\lambda}$ is continuous.

We now specialize to the $B^{*}$-case to extend Theorem 6.11 of [2].

Theorem 4.6. Let $A$ be a $B^{*}$-algebra (with no left ideals of dimension less than three) and let $p$ be any complementor in $A$. Then $p$ is continuous if and only if there is a subsidiary involution \# in $\boldsymbol{A}$ that satisfies $\boldsymbol{R}^{p}=\left(\boldsymbol{R}_{l}\right)^{\#}\left(\boldsymbol{R} \in \boldsymbol{R}_{\boldsymbol{A}}\right)$. If there is such an involution \# then there is an equivalent norm \|\|$^{\prime}$ in $A$ that satisfies the $B^{*}$ condition for \#.

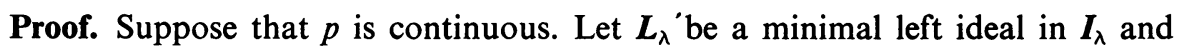
$\langle,\rangle_{\lambda}$ an inner product induced in $L_{\lambda}$ as in Lemma 4.3. Let $T_{a}, T_{a}^{\lambda}$ be the representations described in Theorem 4.4. Consider the restriction to $I_{\lambda}$ of $a \rightarrow T_{a}^{\lambda}$. Since $I_{\lambda}$ is $B^{*}$ and hence its left regular representation on $\boldsymbol{L}_{\lambda}$ is isometric, we have

$$
\|a\| / M<\left|T_{a}^{\lambda} / \boldsymbol{H}_{\lambda}\right|<M\|a\| \quad\left(a \in \boldsymbol{I}_{\lambda}, \lambda \in \Lambda\right) .
$$

Also $p_{\lambda}$ is represented by $\langle,\rangle_{\lambda}$ and it follows that $\boldsymbol{R}^{p_{\lambda}}=\left(\boldsymbol{R}_{l_{\lambda}}\right)^{\#(\lambda)}\left(\boldsymbol{R} \in \boldsymbol{R}_{\boldsymbol{I}_{\lambda}}\right)$ where $\#(\lambda)$ is defined in $\boldsymbol{I}_{\lambda}$ by:

$$
T_{a}^{\lambda} \#(\lambda) \text { is the adjoint of } T_{a}^{\lambda} \text { with respect to }\langle,\rangle_{\lambda}
$$

and $l_{\lambda}$ denotes the left annihilator in $I_{\lambda}$. Now:

$$
\left\|a^{\#(\lambda)}\right\|<M\left|T_{a}^{\lambda} \#(\lambda)\right| \boldsymbol{H}_{\lambda}|=M| T_{a}^{\lambda}\left|\boldsymbol{H}_{\lambda}\right|<M^{2}\|a\| \quad\left(a \in \boldsymbol{I}_{\lambda}, \lambda \in \Lambda\right) .
$$

Since $A$ is $B^{*}, A$ is isometrically $*$-isomorphic with $\left(\sum_{\lambda \in \Lambda} I_{\lambda}\right)_{0}[6$, Theorem 4.10.14] and now since $M$ is independent of $\lambda$ we can define $\#$ on $A$ by $\left(a^{\#}\right)(\lambda)=(a(\lambda))^{\#(\lambda)}$. 
Also $a^{\#} a=0$ implies $a=0$, since in the proof of (iii) $\Rightarrow$ (ii) of [2, Theorem 6.11] it was shown that $(a(\lambda))^{\#(\lambda)} a(\lambda)=0$ implies $a(\lambda)=0$. Hence by Corollary 7.2 and Lemma 7.1 in [2]:

$$
\left(\boldsymbol{R}_{1}\right)^{\#}=\left(\sum_{\lambda \in \Lambda}\left(\boldsymbol{R}_{\lambda}\right)_{l_{\lambda}}^{\#}\right)_{0}=\left(\sum_{\lambda \in \Lambda}\left(\boldsymbol{R}_{\lambda}\right)_{l_{\lambda}}^{\#(\lambda)}\right)_{0}=\left(\sum_{\lambda \in \Lambda} \boldsymbol{R}_{\lambda}^{p_{\lambda}}\right)_{0}=\boldsymbol{R}^{p} \quad\left(\boldsymbol{R} \in R_{A}\right) .
$$

The converse and the remainder of the theorem are contained in Theorem 7.4 of [2].

The operators $E_{\lambda}$. It remains unknown whether in general the operators $E_{\lambda}$ are uniformly bounded. This is true in the case when $A$ is $B^{*}\left(\left|E_{\lambda}\right|=1\right)$ and could have simplified slightly some of the proofs had we been interested only in the $B^{*}$ case. In general, an equivalent question is whether each $a$ in $A$ may be expressed in the form $a=\lim _{n \rightarrow \infty} \sum_{r=1}^{r_{n}} E_{\lambda_{r}} a\left(\left\{\lambda_{r}\right\} \subset \Lambda\right)$.

\section{Two counterexamples.}

1. $C_{2}$ cannot be replaced by $\operatorname{cl}\left(\boldsymbol{R}+\boldsymbol{R}^{p}\right)=\boldsymbol{A}$.

Let $\boldsymbol{H}$ be a three dimensional Hilbert space with an orthonormal basis $\{x, y, z\}$. Let the inner product in $\boldsymbol{H}$ be denoted by (, ). Define an operator $T_{n}$ on $\boldsymbol{H}$ by $T_{n}(\alpha x+\beta y+\gamma z)=n \alpha x+\beta y+\gamma z \quad(\alpha, \beta, \gamma \in C)$. Then $T_{n}$ is a bounded, positive, hermitian operator on $\boldsymbol{H}$ and its inverse is defined and is an operator of the same kind. Thus we may define an equivalent inner product $\langle,\rangle_{n}$ in $\boldsymbol{H}$ by $\langle u, v\rangle_{n}$ $=\left(T_{n} u, v\right)$. Let $\boldsymbol{A}$ be $\boldsymbol{K}(\boldsymbol{H})$ and $p_{n}$ be $p_{\langle\rangle_{n}}$. Now let $\boldsymbol{B}$ be the sum $\left(\sum_{i=1}^{\infty} A_{i}\right)_{0}$ of the countable collection $\left\{\boldsymbol{A}_{i}\right\}$ where $\boldsymbol{A}_{i}=\boldsymbol{A}$ for all $i$. Then $\boldsymbol{B}$ is a dual $\boldsymbol{B}^{*}$-algebra and so, by Lemma 7.1 in [2], $R=\left(\sum_{i=1}^{\infty} \boldsymbol{R}_{i}\right)_{0}\left(\boldsymbol{R} \in R_{B}\right)$ where $\boldsymbol{R}_{i}$ is the intersection of $\boldsymbol{R}$ with the image of $\boldsymbol{A}_{i}$ in $B$. Define $p$ on $B$ by $R^{p}=\left(\sum_{i=1}^{\infty} R_{i}^{p_{i}}\right)_{0}$. Then it can easily be verified that $p$ satisfies $C_{1}, C_{3}, C_{4}$; also $R+R^{p}$ is dense in $B\left(R \in R_{B}\right)$. Suppose that $p$ is a complementor. Then since each $p_{i}$ is continuous, $p$ is continuous. Now it is clear that we may substitute $\boldsymbol{H}$ for $\boldsymbol{L}_{\lambda}$ in Lemma 4.3. Thus, taking the slightly stronger result in the proof of that lemma we see that if $[,]_{n}$ are inner products in $\boldsymbol{H},[,]_{n}$ represents $p_{n}$ and $\|h\|^{2} \leqq[h, h]_{n} \geqq 2\|h\|^{2}(h \in \boldsymbol{H})(\|\|$ denotes the norm in $\boldsymbol{H}$ ), then there exists a finite constant $M$ such that $[h, h]_{n} \leqq M\|h\|^{2}$ for all $h$ in $\boldsymbol{H}$ and all integers $n$. Now $\langle,\rangle_{n}$ is such a collection of inner products. However, $\langle x, x\rangle_{n}=n\|x\|^{2}$ which contradicts the existence of $M$. Therefore $B$ is not complemented.

2. The dimension restriction in Theorems 3.3, 3.4, 4.4, 4.6 cannot be removed.

Let $\boldsymbol{H}$ be a two-dimensional Hilbert space and $\{x, y\}$ an orthonormal basis in $\boldsymbol{H}$. We wish to construct a continuous complementor $p$ in $\boldsymbol{K}=\boldsymbol{K}(\boldsymbol{H})$ such that $\boldsymbol{p}$ is not $p_{\langle>}$for any inner product $\langle,>$in $\boldsymbol{H}$. To do this it is clearly sufficient (from the method of Theorem 3.2) to construct a continuous LSC $q$ in $\boldsymbol{H}$ that is not an orthogonal complementation with respect to any inner product.

Let $\lambda=r e^{i \theta} \in C, \lambda \neq 0$, and let $\lambda^{\prime}$ be defined by

$$
\lambda^{\prime}=-e^{i \theta}\left(a^{2} \cos ^{2} \theta+b^{2} \sin ^{2} \theta\right) / r
$$


where $a, b$ are any two distinct positive reals. Then $\lambda \rightarrow \lambda^{\prime}$ is a continuous, one-toone, involutory map of $C-(0)$ onto $C-(0)$ and it has no fixed points. A geometrical interpretation of the map is as follows:

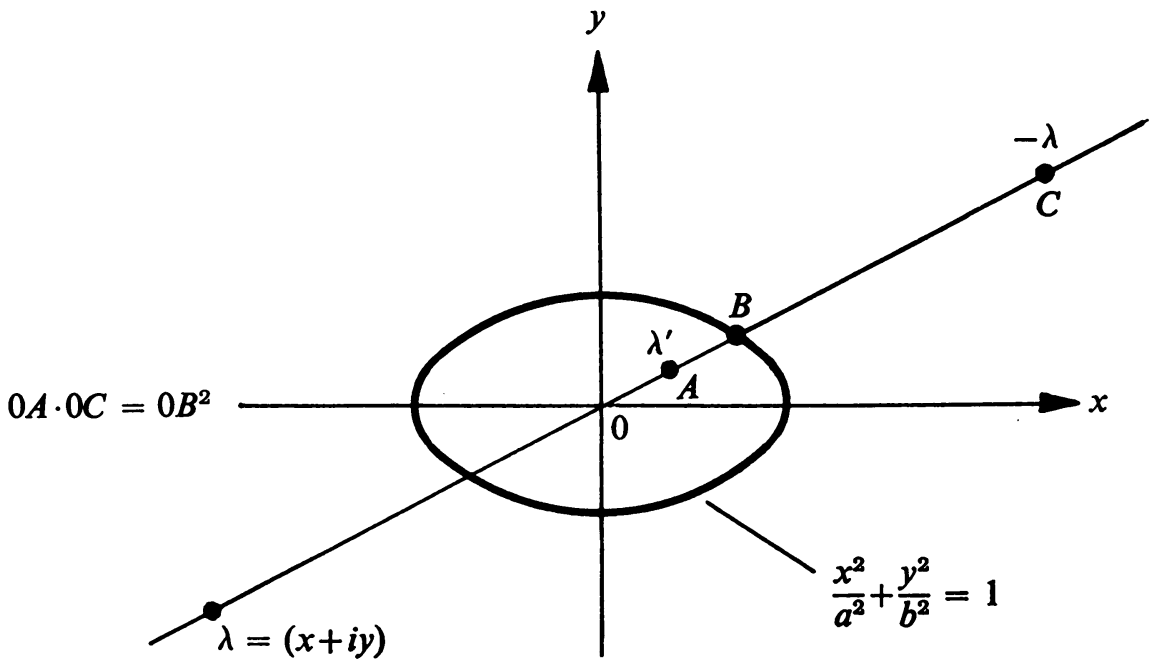

Also, as $\lambda \rightarrow 0, \lambda^{\prime} \rightarrow \infty$ and as $\lambda \rightarrow \infty, \lambda^{\prime} \rightarrow 0$.

Now $q$ can be defined on $\boldsymbol{H}$ by:

$$
[x]^{q}=[y], \quad[y]^{q}=[x], \quad \boldsymbol{H}^{q}=(0),(0)^{q}=\boldsymbol{H}, \quad[x+\lambda y]^{q}=\left[x+\lambda^{\prime} y\right], \quad \lambda \neq 0 .
$$

It is easy to see that $q$ is an LSC. Also:

$f_{x}=x \otimes x, \quad f_{y}=y \otimes y, \quad f_{x+\lambda y}=(x+\lambda y) \otimes\left(x+\lambda^{*} y\right) /\left(x+\lambda^{*} y, x+\lambda y\right)$

$$
\left(\lambda \neq 0, \text { co } \lambda^{*}=-1 / \lambda^{\prime}\right) \text {. }
$$

Then, since $\lambda \rightarrow \lambda^{\prime}$ is continuous, $\lambda \rightarrow \lambda^{*}$ is continuous on $C-(0)$. As $\lambda \rightarrow 0$, $\lambda^{*} \rightarrow 0$, and as $\lambda \rightarrow \infty, \lambda^{*} \rightarrow \infty$. It is now clear that $f_{z_{n}} \rightarrow f_{z}$ when $z_{n} \rightarrow z$ and $z_{n}$, . $z \in H$; thus $q$ is continuous.

Suppose that $q$ is orthogonal complementation with respect to an inner product $\langle$,$\rangle in \boldsymbol{H}$. Then $\langle x, y\rangle=\left\langle x+\lambda y, x+\lambda^{\prime} y\right\rangle=0$. Therefore,

$$
\langle x, x\rangle+\lambda \cos \lambda^{\prime}\langle y, y\rangle=0 \text {. }
$$

Let $\lambda=r e^{i \theta}$ : then $\lambda \operatorname{co} \lambda^{\prime}=-\left(a^{2} \cos ^{2} \theta+b^{2} \sin ^{2} \theta\right) \neq$ constant. Thus (1) cannot be satisfied for all $\lambda$.

\section{REFERENCES}

1. F. E. Alexander, On complemented and annihilator algebras, Glasgow J. Math. 10 (1969), 38-45.

2. F. E. Alexander and B. J. Tomiuk, On complemented $B^{*}$-algebras, Trans. Amer. Math. Soc. 137 (1969), 459-480. 
3. F. F. Bonsall and A. W. Goldie, Annihilator algebras, Proc. London Math. Soc. (3) 4 (1954), 154-167. MR 15, 881.

4. S. Kakutani and G. W. Mackey, Ring and lattice characterizations of complex Hilbert space, Bull. Amer. Math. Soc. 52 (1946), 727-733. MR 8, 31.

5. C. Pearcy and D. M. Topping, Sums of small numbers of idempotents, Michigan Math. J. 14 (1967), 453-465. MR 36 \#2006.

6. C. E. Rickart, General theory of Banach algebras, Van Nostrand, Princeton, N. J., 1960. MR 22 \#5903.

7. A. E. Taylor, Introduction to functional analysis, Wiley, New York, 1958. MR 20 \#5411.

8. B. J. Tomiuk, Structure theory of complemented Banach algebras, Canad. J. Math. 14 (1962), 651-659. MR 26 \#626.

\section{UNIVERSITY OF GLASGOW,}

Glasgow, SCOTLAND 